\title{
Studies on Image Segmentation Integrating Generalized Laplace Mixture Model and Hierarchical Clustering
}

\author{
T. Jyothirmayi \\ Department of CSE \\ GITAM Universit \\ Visakhapatnam
}

\author{
K. Srinivasa Rao \\ Department of \\ Statistics \\ Andhra University \\ Visakhapatnam
}

\author{
P. Srinivasa Rao \\ Department of CS \\ \&SE \\ Andhra University \\ Visakhapatnam
}

\author{
Ch.Satyanarayana \\ Department of CSE \\ JNTU-Kakinada \\ Kakinada
}

\begin{abstract}
In many practical applications such as security and surveillance, robotics, medical diagnostics, remote sensing, video processing the image segmentation plays a dominant role. In general the image segmentation is performed either hierarchical method or model based methods. Both methods have advantages and disadvantages. Integrating these two methods will provide efficient utilization of resources and increases segmentation performance. Hence, in this paper an image segmentation method based on generalized Laplace Mixture Model integrated with hierarchical clustering method was developed and analyzed. The updated equations for estimating the model parameters using EM algorithm are derived for the generalized Laplace Mixture Model for the first time. The segmentation algorithm is presented under component maximum likelihood with Bayesian criteria. The efficiency of the proposed algorithm is validated by selecting sample images from Berkeley image data set and computing the segmentation performance measures such as GCE, PRI and VOI. A comparative study of proposed algorithm with that of model based image segmentation algorithm on GMM revealed that the proposed algorithm outperforms the existing ones.
\end{abstract}

\section{General Terms}

Image segmentation, Gaussian distribution, EM algorithm.

\section{Keywords}

Segmentation, Image Segmentation, Image Analysis, Laplace distribution.

\section{INTRODUCTION}

Image analysis is an important step in image processing which deals with deriving object description from an image. Image segmentation refers to dividing the image into number of regions based on the properties of pixel. The different image segmentation techniques can be categorized into two categories i) Statistical (Dubes R.C and Jain A.K(1998)) ii) Structural(Udapa J.K. et al (1996)). Several researchers have proposed solutions for segmentation problem using the statistical image models (Srinivasa Rao et al(2007)). Hua Yang (2000) investigated a probability model based image segmentation method where it was assumed that image pixel features follow a Gaussian mixture distribution. EM algorithm was used for estimating parameters and initializing parameters. Anisotropic diffusion and histogram analysis methods were applied. Yunjie Chen (2014) studied a model based on GMM and nonlocal information. To obtain a smooth bias field Legendre Polynomials were used to fit it and merged it to the EM framework. Non local information which reduced the effect of noise was also considered. Celia A.(2014) studied soft multi-phase segmentation model where it was assumed that the pixel intensities are distributed as a Gaussian mixture. The model was formulated as a minimization problem making use of the maximum likelihood estimator and phase-transition theory. The mixture coefficients, which were estimated using a spatially varying mean and variance procedure, were used for image segmentation.

Shanaz Aman et al (2015) implemented HMRF-EM (Hidden Markov Random Field-EM) assuming the image as 2D gray level and intensities of pixel follow Gaussian distribution and same was implemented for color image segmentation. Vamsi Krishna M (2015) developed an image segmentation technique based on bivariate Gaussian distribution.. Srinivasa Rao et al (2012) applied a symmetric mixture model combined with hierarchical clustering for symmetric images. It is proved that their method works better than the method with GMM. Rajkumar G.V.S et al (2011) developed an image segmentation algorithm using finite mixture of doubly truncated bivariate Gaussian distribution by integrating with hierarchical clustering.

However, the major drawbacks of GMM are that it assumes that the pixel intensities are mesokurtic and symmetrically distributed in each image region. But, in many images the pixel intensities may not be distributed as mesokurtic even though symmetry is maintained. This deviation in segmenting images has reduced the performance of image segmentation algorithms based on GMM. To improve the efficiency of image segmentation method one has to consider the generalization of the GMM with respect to kurtosis associated with pixel intensities in each image region. An alternative to Gaussian distribution with respect to platykurtic variates is served by a Laplace probability model (Norman L.Johnson, Samuel Kotz and Balakrishnan (1994)). The Laplace distribution is further generalized by Srinivasa Rao et al(1997) and named it as generalized Laplace distribution. This generalized Laplace distribution includes a spectrum of platykurtic distributions for which pixel intensities in image region are having kurtosis less than three for specific values of indexing parameter.

Very little work has been reported in literature in image segmentation algorithm based on mixture of generalized Laplace distributions which will provide accurate performance in image segmentation for images which are having platykurtic nature of pixel intensity distribution in image regions. With this motivation in this paper an image segmentation algorithm is developed and analyzed with mixture of generalized Laplace distribution integrated with hierarchical clustering. In this paper the experimentation is done on images obtained from Berkeley data set (www.eecs.berkeley.edu). The performance of algorithm is evaluated by calculating PRI (probability Random Index), VOI (Variation of Information) and GCE(Global Consistency Error). The images are reconstructed using maximum likelihood estimates and probability model. The reconstructed 
images performance is evaluated by calculating quality metrics such as Average difference, Maximum distance, Image fidelity, Mean Square Error and Signal Noise ratio. The remaining paper is organized as follows section 2 deals with Mixture of Generalized Laplace distribution, section 3 deals with estimation of model parameters through EM algorithm, Section 4 explains about updating initial estimates and section 5 describes the image segmentation algorithm. The experimentation and results are presented in section 6 and finally conclusion in section 7 .

\section{GENERALIZED LAPLACE MODEL AND MIXTURE OF GENERALIZED LAPLACE MODEL}

In image analysis the entire image is considered as a union of several image regions. In each image region the image data is quantized by pixel intensities. For a given point pixel $(x, y)$, the pixel intensity $\mathrm{z}=\mathrm{f}(\mathrm{x}, \mathrm{y})$ is a random variable. To model the pixel intensities of the image region it is assumed that the pixel intensities within the region follow a generalized Laplace distribution. The probability density function of the pixel intensity is given by

$f(x, \mu, \sigma 2)=\left(\frac{\left(r^{2}+\frac{(x-\mu)^{2}}{\sigma^{2}}\right)^{r} e^{-\left|\frac{x-\mu}{\sigma}\right|}}{2 \sigma \sum_{k=0}^{r}\left(\begin{array}{l}r \\ k\end{array}\right)^{2(r-k)}(2 k !)}\right)$ where $-\infty<\mathbf{x}<\infty,-$

$\infty<\mu<\infty, \sigma>0$

The probability curve of the distribution is presented in figure

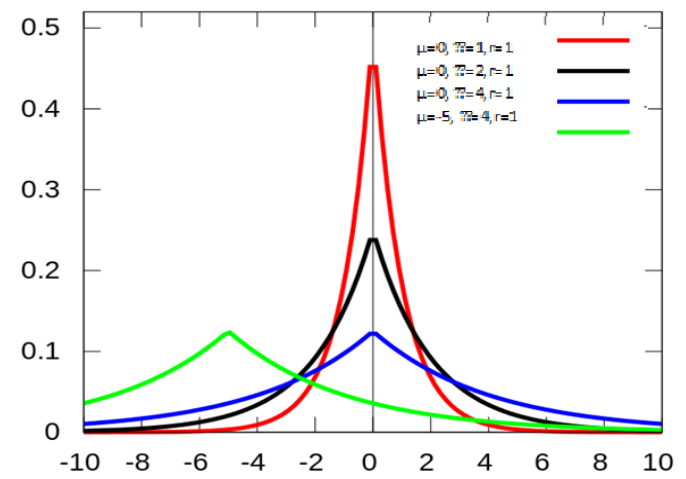

Fig 1: Frequency Curve

The mean value of pixel intensity $\mathrm{X}$ is obtained as $\mathrm{E}[\mathrm{X}]=\mu$,

Variance $\mathrm{V}(\mathrm{X})=\mathrm{E}\left[(\mathrm{x}-\mu)^{2}\right]=2 \sigma^{2}$.

It is a symmetric distribution.Here, it is assumed that the entire image is a collection of several image regions. In each image region the pixel intensities are characterized by generalized laplace probability model. As a result of which the pixel intensities in the whole image region is characterized by a k-component mixture of generalized laplace distribution. The probability density function of pixel intensities in whole image is of the form

$p(x)=\sum_{i=1}^{k} \alpha_{i} f_{i}\left(\mathrm{x}_{\mathrm{s}}, \mu_{i}, \sigma_{i}^{2}\right)$,

where $\mathrm{k}$ is the number of regions, $0 \leq \alpha_{\mathrm{i}} \leq 1$ are weights such that $\Sigma \alpha_{\mathrm{i}}=1$ and $\alpha_{\mathrm{i}}$ is the weight associated with $\mathrm{i}^{\text {th }}$ region in the whole image and $f\left(x, \mu, \sigma^{2}\right)$ is the probability density function of Generalized Laplace distribution and is as given in equation(1)Usually the pixel intensities in image regions are correlated. The spacial sampling method or spacial averaging methods are applied for reducing the correlation among pixel intensities (Lie.T and Sewehand. W(1992)) (Kelly P.A.et $\mathrm{al}(1998)$ ). The mean pixel intensity of the whole image region is

$E(Z)=\sum_{i=1}^{k} \alpha_{i} \mu_{i}$

\section{ESTIMATION OF THE MODEL PARAMETERS BY EM ALGORITHM}

The estimates of the model parameters are obtained through EM algorithm. It is assumed that the intensity of pixel in image region follows a new Laplace distribution and whole image is characterized with a finite mixture of new generalized Laplace distributions. Its probability distribution function is given in equation (2).

The likelihood function of observations $\mathrm{x}_{1}, \mathrm{x}_{2} \ldots \mathrm{x}_{\mathrm{n}}$ is

$$
\begin{aligned}
& \mathrm{L}(\theta)=\prod_{s=1}^{N} p\left(\mathrm{x}_{\mathrm{s}}, \theta^{l}\right) \\
& \mathrm{L}(\theta)=\prod_{s=1}^{N}\left(\sum_{i=1}^{k} \alpha_{i} f_{i}\left(\mathrm{x}_{\mathrm{s}}, \mu_{i}, \sigma_{i}{ }^{2}\right)\right)
\end{aligned}
$$

This implies

$\log \mathrm{L}(\theta)=\sum_{s=1}^{N} \log \left(\sum_{i=1}^{k} \alpha_{i} f_{i}\left(\mathrm{x}_{\mathrm{s}}, \mu_{i}, \sigma_{i}^{2}\right)\right) \quad$ where $\theta=\left\{\mu, \sigma^{2}, \alpha_{i} ; \mathrm{i}=1,2, . . \mathrm{k}\right\}$

$\sum_{s=1}^{N} \log \left[\left(\frac{\left(r^{2}+\frac{(x-\mu)^{2}}{\sigma^{2}}\right)^{r} e^{-\left|\frac{x-\mu}{\sigma}\right|}}{2 \sigma \sum_{k=0}^{r}\left(\begin{array}{l}r \\ k\end{array}\right) r^{2(r-k)(2 k !)}}\right)\right]$

E Step: In the E Step the expectation value of $\log \mathrm{L}(\theta)$ with respect to initial parameter $\theta^{0}$ is

$\mathrm{Q}\left(\theta ; \theta^{0}\right)=E_{\theta^{0}}[\log L(\theta)]$

$\mathrm{P}\left(x_{s}, \theta^{\mathrm{l}}\right)=\sum_{\mathrm{i}=1}^{\mathrm{k}} \alpha_{\mathrm{i}}^{\mathrm{l}} \mathrm{f}_{\mathrm{i}}\left(\mathrm{x}_{\mathrm{s}}, \theta^{\mathrm{l}}\right)$

$\log \mathrm{L}(\theta)=\sum_{\mathrm{s}=1}^{\mathrm{N}} \log \left(\sum_{\mathrm{i}=1}^{\mathrm{k}} \alpha_{\mathrm{i}}^{\mathrm{l}} \mathrm{f}_{\mathrm{i}}\left(\mathrm{x}_{\mathrm{s}}, \theta^{\mathrm{l}}\right)\right.$

The conditional probability of $\mathrm{x}_{\mathrm{s}}$ belonging to region $\mathrm{k}$ is

$\mathrm{T}_{\mathrm{k}}\left(\mathrm{x}_{\mathrm{s}}, \theta^{\mathrm{l}}\right)=\frac{\alpha_{\mathrm{k}} \mathrm{l}_{\mathrm{k}}\left(\mathrm{x}_{\mathrm{s}}, \theta^{\mathrm{l}}\right)}{\mathrm{p}\left(\mathrm{x}_{\mathrm{s}}, \theta^{1}\right)}=\frac{\alpha_{\mathrm{k}}{ }^{\mathrm{l}} \mathrm{f}_{\mathrm{k}}\left(\mathrm{x}_{\mathrm{s}}, \theta^{\mathrm{l}}\right)}{\sum_{\mathrm{i}=1}^{\mathrm{k}} \mathrm{\alpha}_{\mathrm{i}} \mathrm{f}_{\mathrm{i}}\left(\mathrm{x}_{\mathrm{s}}, \theta^{\mathrm{l}}\right)}$

$\mathrm{Q}\left(\theta ; \theta^{0}\right)=\sum_{\mathrm{i}=1}^{\mathrm{k}} \sum_{\mathrm{s}=1}^{\mathrm{N}} \mathrm{T}_{\mathrm{i}}\left(\mathrm{x}_{\mathrm{s}}, \theta^{\mathrm{l}}\right)\left(\log \mathrm{f}_{\mathrm{i}}\left(\mathrm{x}_{\mathrm{s}}, \theta^{\mathrm{l}}\right)+\log \alpha_{\mathrm{i}}^{\mathrm{l}}\right.$

where, $\mathrm{f}_{\mathrm{i}}\left(\mathrm{x}_{\mathrm{S}}, \theta^{\mathrm{l}}\right)=\left(\frac{\left(r^{2}+\frac{(x-\mu)^{2}}{\sigma^{2}}\right)^{r} e^{-\left|\frac{x-\mu}{\sigma}\right|}}{2 \sigma \sum_{k=0}^{r}\left(\begin{array}{l}r \\ k\end{array}\right) r^{2(r-k)}(2 k !)}\right)$

M Step: To get estimation of parameters, maximize $\mathrm{Q}\left(\theta ; \theta^{l}\right)$ such that $\sum \alpha_{\mathrm{i}}=1$

Using Lagrange type function and maximizing

$\widehat{\alpha_{i}}=\frac{1}{\mathrm{~N}} \sum_{\mathrm{s}=1}^{\mathrm{N}} \mathrm{T}_{\mathrm{i}}\left(\mathrm{x}_{\mathrm{s}}, \theta^{\mathrm{l}}\right)$

$\alpha_{\mathrm{i}}$ for $(1+1)$ th iteration is

$\alpha_{\mathrm{i}}^{\mathrm{l}+1}=\frac{1}{\mathrm{~N}} \sum_{\mathrm{s}=1}^{\mathrm{N}} \mathrm{T}_{\mathrm{i}}\left(\mathrm{x}_{\mathrm{s}}, \theta^{\mathrm{l}}\right)=$

$\frac{1}{N} \sum_{s=1}^{N}\left[\frac{\alpha_{i}^{1} f_{i}\left(x_{s}, \theta^{l}\right)}{\sum_{i=1}^{k} \alpha_{i}^{1} f_{i}\left(x_{s}, \theta^{1}\right)}\right]$

For updating the parameter $\mu_{\mathrm{i}}, \mathrm{i}=1,2, . . \mathrm{k}$,

Consider the derivative of $\mathrm{Q}\left(\theta ; \theta^{l}\right)$ with respect to $\mu_{\mathrm{i}}$, and equate to 0

$\mathrm{Q}\left(\theta ; \theta^{l}\right)=\mathrm{E}\left[\log \mathrm{L}\left(\left(\theta ; \theta^{l}\right)\right]\right.$ 


$$
\frac{\partial}{\partial \mu \mathrm{i}} \mathrm{Q}\left(\theta ; \theta^{l}\right)=0 \text { This implies }
$$

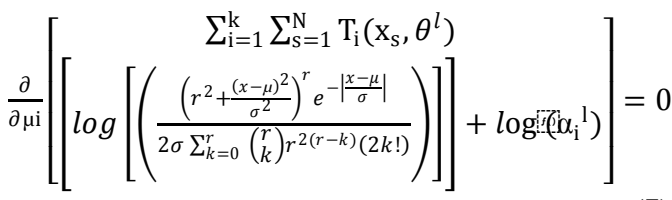

This implies

$$
\frac{\partial}{\partial \mu \mathrm{i}}\left[\sum _ { \mathrm { i } = 1 } ^ { \mathrm { k } } \sum _ { \mathrm { s } = 1 } ^ { \mathrm { N } } \mathrm { T } _ { \mathrm { i } } ( \mathrm { x } _ { \mathrm { s } } , \theta ^ { l } ) \left[\log \left[\left(\left(r^{2}+\frac{\left(\mathrm{x}_{\mathrm{s}}-\mu_{\mathrm{i}}\right)^{2}}{\sigma_{i}{ }^{2}}\right)^{r}\right)\right]-\left|\frac{x_{s}-\mu_{\mathrm{i}}}{\sigma_{i}}\right|-\right.\right.
$$$$
\log 2 \sigma i-\log (k=0 r r k r 2 r-k(2 k !)+\log (\alpha)(\alpha \mathrm{il})=0
$$

This implies

$\sum_{\mathrm{s}=1}^{\mathrm{N}} \mathrm{T}_{\mathrm{i}}\left(\mathrm{x}_{\mathrm{s}}, \theta^{\mathrm{l}}\right)\left[\left(\frac{\left(\mathrm{x}_{\mathrm{s}}-\mu_{\mathrm{i}}\right)}{\sigma_{i}{ }^{2}}\right)\left[\frac{\left[-2 \sigma_{i}{ }^{2}\right]}{\mathrm{r}^{2} \sigma_{i}{ }^{2}+\left(\mathrm{x}_{\mathrm{s}}-\mu_{\mathrm{i}}\right)^{2}}+\frac{\sigma_{\mathrm{i}}}{\left|\mathrm{x}_{\mathrm{s}}-\mu_{\mathrm{i}}\right|}\right]\right]=0$

This implies

$\sum_{\mathrm{s}=1}^{\mathrm{N}} \mathrm{T}_{\mathrm{i}}\left(\mathrm{x}_{\mathrm{s}}, \theta^{\mathrm{l}}\right) \frac{\mathrm{x}_{\mathrm{s}}-\mu_{\mathrm{i}}}{\sigma_{\mathrm{i}}\left|\mathrm{x}_{\mathrm{s}}-\mu_{\mathrm{i}}\right|}-\sum_{s=1}^{N} \mathrm{~T}_{\mathrm{i}}\left(\mathrm{x}_{\mathrm{s}}, \theta^{\mathrm{l}}\right) \frac{\left[2\left(\mathrm{x}_{\mathrm{s}}-\mu_{\mathrm{i}}\right)\right]}{\mathrm{r}^{2} \sigma_{i}{ }^{2}+\left(\mathrm{x}_{\mathrm{s}}-\mu_{\mathrm{i}}\right)^{2}}=0(9)$

where $\mathrm{T}_{\mathrm{i}}\left(\mathrm{x}_{\mathrm{S}}, \theta^{\mathrm{l}}\right)=\frac{\mathrm{a}_{\mathrm{i}}^{\mathrm{l}+1} \mathrm{f}_{\mathrm{i}}\left(\mathrm{x}_{\mathrm{s}}, \theta^{\mathrm{l}}\right)}{\sum_{\mathrm{i}=1}^{\mathrm{k}} \mathrm{a}_{\mathrm{i}}^{1+1} \mathrm{f}_{\mathrm{i}}\left(\mathrm{x}_{\mathrm{s}}, \theta^{\mathrm{l}}\right)}$

For updating $\sigma_{i}^{2}$ differentiate Q $\left(\theta ; \theta^{l}\right)$ w.r.t $\sigma_{i}^{2}$ and equate to 0

$$
\begin{aligned}
& \frac{\partial}{\partial \sigma_{i}{ }^{2}} \mathrm{Q}\left(\theta ; \theta^{l}\right)=0 \\
& \frac{\partial}{\partial \sigma_{i}{ }^{2}}\left[\sum_{\mathrm{i}=1}^{\mathrm{k}} \sum_{\mathrm{S}=1}^{\mathrm{N}} \mathrm{T}_{\mathrm{i}}\left(\mathrm{x}_{\mathrm{S}}, \theta^{l}\right)\left[\log \left[\left(\frac{\left(r^{2}+\frac{\left(x-\mu_{\mathrm{i}}\right)^{2}}{\sigma^{2}}\right)^{r} e^{-\frac{x-\mu_{\mathrm{i}}}{\sigma} \mid}}{2 \sigma \sum_{k=0}^{r}\left(\begin{array}{l}
r \\
k
\end{array}\right) r^{2(r-k)}(2 k !)}\right)\right]\right]\right. \\
& \log (\alpha \mathrm{il})=0
\end{aligned}
$$

This implies

$$
\begin{aligned}
& \frac{\partial}{\partial \sigma_{i}{ }^{2}}\left[\sum _ { \mathrm { i } = 1 } ^ { \mathrm { k } } \sum _ { \mathrm { s } = 1 } ^ { \mathrm { N } } \mathrm { T } _ { \mathrm { i } } ( \mathrm { x } _ { \mathrm { s } } , \theta ^ { l } ) \left[\log \left[\left(\left(r^{2}+\frac{\left(\mathrm{x}_{\mathrm{s}}-\mu_{\mathrm{i}}\right)^{2}}{\sigma_{i}{ }^{2}}\right)^{r}\right)\right]-\right.\right. \\
& x s^{-}-\mu \mathrm{i} \sigma i^{-}-\log 2 \sigma i^{-} \log \log (k=0 r r k r 2 r-k(2 k !)+\log (\alpha \mathrm{il})=0
\end{aligned}
$$

This implies

$$
\sum_{\mathrm{s}=1}^{\mathrm{N}}\left[\frac{\left(\mathrm{x}_{\mathrm{s}}-\mu_{\mathrm{i}}\right)^{2}}{\left(r^{2} \sigma_{i}{ }^{2}+\left(\mathrm{x}_{\mathrm{s}}-\mu_{\mathrm{i}}\right)^{2}\right) \sigma_{i}{ }^{2}}-\left|\frac{x_{s}-\mu_{\mathrm{i}}}{2 \sigma_{i}{ }^{3}}\right|-\frac{1}{2 \sigma_{i}{ }^{2}}\right] \mathrm{T}_{\mathrm{i}}\left(\mathrm{x}_{\mathrm{s}}, \theta^{\mathrm{l}}\right)=0
$$

where, $T_{i}\left(x_{s}, \theta^{l}\right)=\frac{\alpha_{i}^{1+1} f_{i}\left(x_{s}, \theta^{l}\right)}{\sum_{i=1}^{k} \alpha_{i}^{1+1} f_{i}\left(x_{s}, \theta^{1}\right)}$

\section{INITIALIZATION OF THE PARAMETERS BY HIERARCHICAL CLUSTERING:}

For EM algorithm the parameter $\alpha_{\mathrm{i}}$ and the model parameters $\mu_{\mathrm{i}}$ and $\sigma_{\mathrm{i}}^{2}$ should be initialized. The initial values can be taken as $\alpha_{\mathrm{i}}=\frac{1}{\mathrm{k}}$ where $\mathrm{k}$ is the number of image regions obtained from the hierarchical clustering algorithm. The steps involved in hierarchical clustering algorithm are discussed by (S.C.Johnson(1967)). The shape parameter $r$ can be estimated by sample kutosis using the following equation

$\frac{\mu_{4}}{\mu_{2}{ }^{2}}=\frac{\left[\sum_{\mathrm{k}=0}^{\mathrm{r}} \frac{\mathrm{r} !}{\mathrm{k} !(\mathrm{r}-\mathrm{k}) !} \mathrm{r}^{2(\mathrm{r}-\mathrm{k})}(2 \mathrm{k}+4) !\right]\left[\sum_{\mathrm{k}=0}^{\mathrm{r}} \frac{\mathrm{r} !}{\mathrm{k} !(\mathrm{r}-\mathrm{k}) !} \mathrm{r}^{2(\mathrm{r}-\mathrm{k})}(2 \mathrm{k}) !\right]}{\mathrm{x} \sum_{\mathrm{k}=0}^{\mathrm{r}} \frac{\mathrm{r} !}{\mathrm{k} !(\mathrm{r}-\mathrm{k}) !} \mathrm{r}^{2(\mathrm{r}-\mathrm{k})}(2 \mathrm{k}+4) !}$

There exists one root for the shape parameter $r$ in $(0,2 r)$ which is obtained by Newton Raphson Method. Obtaining this shape parameter $\mathrm{r}_{\mathrm{i}}$ the other estimates $\mu_{i}$ and $\sigma_{i}{ }^{2}$ can be obtained by method of moments as

$\widehat{\mu_{i}}=\bar{x}$

$\widehat{\sigma_{i}^{2}}=\left[\frac{\left[\sum_{k=0}^{r} \frac{r !}{k !(r-k) !} r^{2(r-k)}(2 k) !\right]}{\left[\sum_{k=0}^{r} \frac{r !}{k !(r-k) !} r^{2(r-k)}(2 k+2) !\right]}\right] \mathrm{S}^{2}$

where $\mathrm{s}^{2}$ is the sample variance.

Once the initial estimates are obtained, final refined estimates are obtained through EM algorithm given in section 3.

\section{SEGMENTATION ALGORITHM}

After refining the parameters the next step is to segment the image by allocating the pixels to the segments. This operation is performed by segmentation algorithm. The image segmentation algorithm consists of four steps

Step 1: Obtain the number of image regions using hierarchical clustering algorithm.

Step 2: Obtain the initial estimates of the model parameters using hierarchical clustering.

Step 3: Obtain the refined estimates of the model parameters $\alpha_{i}, \mu_{i}, \sigma_{i}^{2}$ for $\mathrm{i}=1,2 \ldots . \mathrm{k}$, by using EM algorithm with updated equations.

Step 4: Assign each pixel into the corresponding $j^{\text {th }}$ region according to the maximum likelihood of the $\mathrm{j}^{\text {th }}$ component $\mathrm{L}_{\mathrm{j}}$.

That is $Z_{s}$ is assigned to the $j^{\text {th }}$ region for which $L$ is maximum.

$$
L=\max \left(\frac{\left(r^{2}+\frac{(x-\mu)^{2}}{\sigma^{2}}\right)^{r} e^{-\left|\frac{x-\mu}{\sigma}\right|}}{2 \sigma \sum_{k=0}^{r}\left(\begin{array}{l}
r \\
k
\end{array}\right) r^{2(r-k)}(2 k !)}\right)
$$

\section{EXPERIMENTATION AND RESULTS}

For experimental analysis five images are randomly selected from Berkeley image dataset (www.eecs.berkeley.edu/Research/Projects/CS/vision/bsds/B SDS300/html/dataset/images.html). The image pixel intensities are taken as feature of the image assuming that they follow mixture of generalized Laplace distribution. The value of $\mathrm{K}$ (number of image regions) is obtained by histogram of pixel intensities. The five images and their respective histograms are shown in figure 2 . 


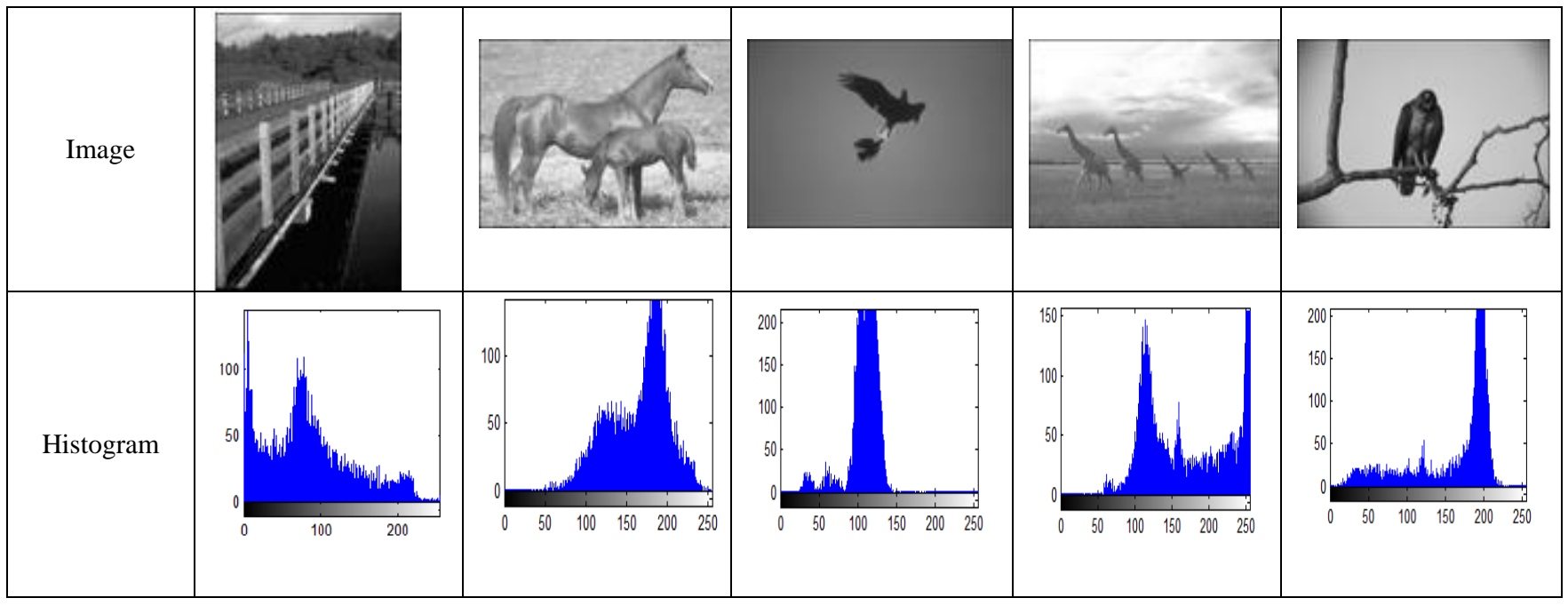

Fig 2: Images and their Histograms

Based on histograms the initial estimates for five images have been obtained. The regions obtained from the image are named as $\mathrm{C}_{1}, \mathrm{C}_{2}, \mathrm{C}_{3} . . \mathrm{C}_{\mathrm{K}}$ where $\mathrm{K}$ is the number of regions. The model parameters considered are $\alpha_{\mathrm{i}}, \mu_{\mathrm{i}}$ and $\sigma_{\mathrm{i}}$ for $\mathrm{i}=1,2 \ldots \mathrm{K}$ and they are obtained by the method given in section 4 . Final parameters for the five images have been derived using these initial parameters and updated equations in section 3 the final parameters and presented in tables 1,2,3,4 and 5 .

Table 1

\begin{tabular}{|c|c|c|c|c|c|c|}
\hline \multicolumn{3}{|c|}{ Estimated Values of the Parameters for Image 1 } \\
Number of regions $(\mathrm{K}=3)$
\end{tabular}

Table 2

\begin{tabular}{|c|c|c|c|c|}
\hline \multicolumn{5}{|c|}{ Estimated Values of the Parameters for the Image2 } \\
Number of regions(K=2)
\end{tabular}

Table 3

Estimated Values of the Parameters for Image3

Number of regions $(\mathrm{K}=3)$

\begin{tabular}{|c|c|c|c|c|c|c|}
\hline \multirow{2}{*}{ Parameters } & \multicolumn{3}{|c|}{$\begin{array}{c}\text { Estimation of Initial } \\
\text { Parameters } \\
\text { By Hierarchical }\end{array}$} & \multicolumn{3}{c|}{$\begin{array}{c}\text { Estimation of Final } \\
\text { Parameters by EM } \\
\text { Algorithm }\end{array}$} \\
\cline { 2 - 7 } & $\mathrm{C}_{1}$ & $\mathrm{C}_{2}$ & $\mathrm{C}_{3}$ & $\mathrm{C}_{1}$ & $\mathrm{C}_{2}$ & $\mathrm{C}_{3}$ \\
\hline$\alpha_{\mathrm{i}}$ & 0.33 & 0.33 & 0.33 & -0.73 & 0.90 & 0.83 \\
\hline$\mu_{\mathrm{i}}$ & 53.46 & 180.85 & 113.02 & 118.31 & 104.94 & 48.80 \\
\hline$\sigma_{\mathrm{i}}$ & 16.03 & 4.94 & 11.2 & 13.82 & 2.93 & 15.8 \\
\hline
\end{tabular}

Table 4

\begin{tabular}{|c|c|c|c|c|c|c|}
\hline \multicolumn{8}{|c|}{ Estimated Values of the Parameters for Image4 } \\
Number of regions(K=3) \\
\hline \multirow{2}{*}{$\begin{array}{c}\text { Paramete } \\
\text { rs }\end{array}$} & \multicolumn{3}{c|}{$\begin{array}{c}\text { Estimation of Initial } \\
\text { Parameters } \\
\text { By Hierarchical }\end{array}$} & \multicolumn{2}{c|}{$\begin{array}{c}\text { Estimation of Final } \\
\text { Parameters by EM } \\
\text { Algorithm }\end{array}$} \\
\cline { 2 - 7 } & $\mathrm{C}_{1}$ & $\mathrm{C}_{2}$ & $\mathrm{C}_{3}$ & $\mathrm{C}_{1}$ & $\mathrm{C}_{2}$ & $\mathrm{C}_{3}$ \\
\hline$\alpha_{\mathrm{i}}$ & 0.33 & 0.33 & 0.33 & -0.16 & 1.15 & 0.015 \\
\hline$\mu_{\mathrm{i}}$ & 242.7 & 34.3 & 179.7 & 190.3 & 30.3 & 170.8 \\
\hline$\sigma_{\mathrm{i}}$ & 0 & 3 & 9 & 5 & 8 & 0 \\
\hline
\end{tabular}

Table 5

\begin{tabular}{|c|c|c|c|c|c|c|}
\hline \multicolumn{6}{|c|}{ Estimated Values of the Parameters for Image 5 } \\
Number of regions(K=3) \\
\hline \multirow{3}{*}{$\begin{array}{c}\text { Parameter } \\
\mathrm{s}\end{array}$} & \multicolumn{3}{|c|}{$\begin{array}{c}\text { Estimation of Initial } \\
\text { Parameters } \\
\text { By Hierarchical }\end{array}$} & $\begin{array}{c}\text { Estimation of Final } \\
\text { Parameters by EM } \\
\text { Algorithm }\end{array}$ \\
\cline { 2 - 7 } & $\mathrm{C}_{1}$ & $\mathrm{C}_{2}$ & $\mathrm{C}_{3}$ & $\mathrm{C}_{1}$ & $\mathrm{C}_{2}$ & $\mathrm{C}_{3}$ \\
\hline$\alpha_{\mathrm{i}}$ & 0.33 & 0.33 & 0.33 & 0.25 & -0.09 & 0.83 \\
\hline$\mu_{\mathrm{i}}$ & 110.9 & 190.5 & 51.0 & 60.3 & 164.3 & 74.8 \\
& 1 & 3 & 8 & 5 & 8 & 0 \\
\hline
\end{tabular}




\begin{tabular}{|c|c|c|c|c|c|c|}
\hline$\sigma_{\mathrm{i}}$ & 14.39 & 14.82 & $\begin{array}{c}18.6 \\
2\end{array}$ & $\begin{array}{c}18.5 \\
3\end{array}$ & 12.50 & $\begin{array}{c}34.3 \\
9\end{array}$ \\
\hline
\end{tabular}

Substituting the final estimates of the model parameters the probability density function of pixel intensities of each image are estimated.The estimated probability density function of the pixel intensities of the image 1 is $\mathrm{f}\left(\mathrm{x}, \theta^{\mathrm{l}}\right)=$

$$
\begin{gathered}
\left(\frac{1}{140.52}\right)\left(1+\frac{(x-56.99)^{2}}{23.42^{2}}\right)^{r} e^{-\left|\frac{x-56.99}{23.42}\right|}+ \\
\left(\frac{1}{170.58}\right)\left(1+\frac{(x-6.84)^{2}}{8.43}\right)^{r} e^{-\left|\frac{x-6.84}{8.43}\right|}+ \\
(1 / 1501.98)\left(1+\frac{(x-165.87)^{2}}{25.33^{2}}\right)^{r} e^{-\left|\frac{x-165.87}{25.33}\right|}
\end{gathered}
$$

The estimated probability density function of the pixel intensities of image 2 is $f\left(x, \theta^{1}\right)=$

$$
\begin{aligned}
& \left(\frac{1}{137.64}\right)\left(1+\frac{(x-92.64)^{2}}{22.94^{2}}\right)^{r} e^{-\left|\frac{x-92.64}{22.94}\right|} \\
& +(1 / 122.94)\left(1+\frac{(x-78.77)^{2}}{20.49^{2}}\right)^{r} e^{-\left|\frac{x-78.77}{20.49}\right|}
\end{aligned}
$$

The estimated probability density function of the pixel intensities of image 3 is $f\left(x, \theta^{l}\right)=$

$$
\begin{aligned}
& \left(\frac{1}{82.92}\right)\left(1+\frac{(x-118.31)^{2}}{13.82^{2}}\right)^{r} e^{-\left|\frac{x-118.31}{13.82}\right|} \\
+ & \left(\left(\frac{1}{167.58}\right)\right)\left(1+\frac{(x-104.94)^{2}}{27.93^{2}}\right)^{r} e^{-\left|\frac{x-104.94}{27.93}\right|} \\
+ & \left.(1 / 94.8)\left(1+\frac{(x-48.80)^{2}}{15.82^{2}}\right)^{r} e^{-\left|\frac{x-48.30}{15.82}\right|}\right)
\end{aligned}
$$

The estimated probability density function of the pixel intensities of image 4 is $\mathrm{f}\left(\mathrm{x}, \theta^{\mathrm{l}}\right)=$

$$
\begin{gathered}
\left(\frac{1}{57.18}\right)\left(1+\frac{(x-190.35)^{2}}{9.53^{2}}\right)^{r} e^{-\left|\frac{x-190.35}{9.53}\right|}+ \\
\left(\frac{1}{21}\right)\left(1+\frac{(x-30.38)^{2}}{3.5^{2}}\right)^{r} e^{-\left|\frac{x-30.38}{3.5}\right|}+ \\
(1 / 164.34)\left(1+\frac{(x-170.80)^{2}}{27.39^{2}}\right)^{r} e^{-\left|\frac{x-170.80}{27.39}\right|}
\end{gathered}
$$

The estimated probability density function of the pixel intensities of image 5 is $f\left(x, \theta^{l}\right)=$

$$
\begin{aligned}
& \left(\frac{1}{111.18}\right)\left(1+\frac{(x-60.35)^{2}}{18.53^{2}}\right)^{r} e^{-\left|\frac{x-60.35}{18.53}\right|}+ \\
& \left(\frac{1}{75}\right)\left(1+\frac{(x-164.38)^{2}}{12.50^{2}}\right)^{r} e^{-\left|\frac{x-164.38}{12.50}\right|} \\
& +(1 / 206.34)\left(1+\frac{(x-74.80)^{2}}{34.39^{2}}\right)^{r} e^{-\left|\frac{x-74.80}{34.39}\right|}
\end{aligned}
$$

Using the estimated probability density function and segmentation algorithm in section 5, image segmentation is performed for the five images. The original images and segmented images are shown in figure 3.

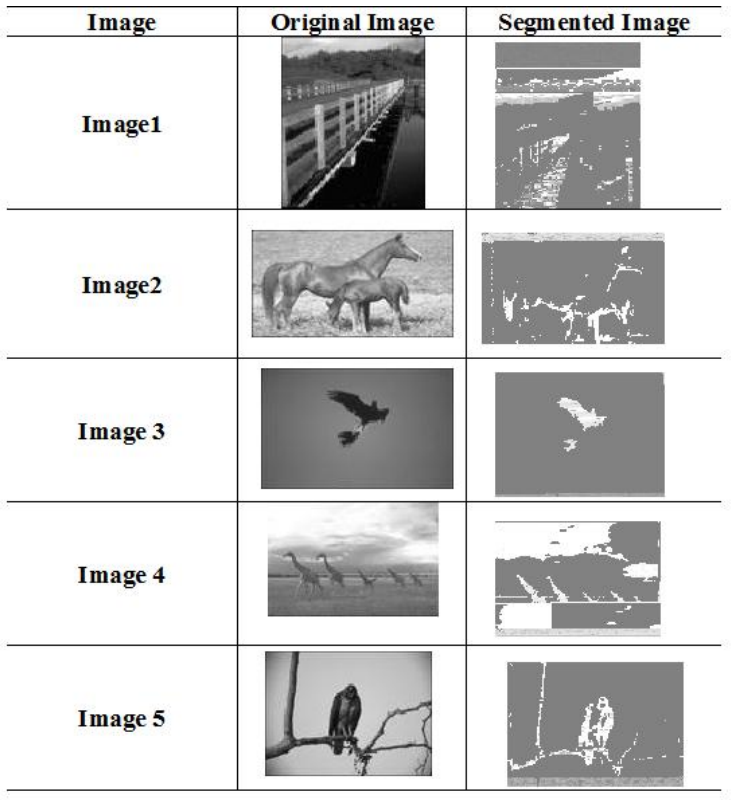

Fig 3: Original and Segmented Images

\section{PERFORMANCE MEASURES}

To evaluate the performance of image segmentation algorithm, performance metrics like probabilistic rand index (PRI) given by Unnikrishnan R. et.al (2007), global consistency error(GCE) given by Martin D. and et.al (2001) and variation of information (VOI) given by Meila M.(2005) have been calculated and compared with existing GMM. The standard criterion for the metrics is that PRI and GCE values must lie in range 0 to 1 while VOI can be as big as possible. The computed values of segmentation performance measure for segmentation methods based on Generalized Laplace Mixture Model using Hierarchical and GMM are given in Table 6.

Table 6: Segmentation Performance Measures

\begin{tabular}{|c|c|c|c|c|}
\hline \multirow{2}{*}{ Image } & \multirow{2}{*}{ Method } & \multicolumn{3}{|c|}{ Performance Measures } \\
\cline { 3 - 5 } Image1 & GMM & 0.94 & 0.72 & 2.84 \\
\cline { 2 - 5 } & GLMM-H & 0.99 & 0.68 & 2.25 \\
\hline \multirow{2}{*}{ Image2 } & GMM & 0.96 & 0.78 & 2.55 \\
\cline { 2 - 5 } & GLMM-H & 0.98 & 0.75 & 2.23 \\
\hline \multirow{2}{*}{ Image3 } & GMM & 0.97 & 0.77 & 2.32 \\
\cline { 2 - 5 } & GLMM-H & 0.98 & 0.75 & 1.48 \\
\hline \multirow{3}{*}{ Image 4 } & GMM & 0.97 & 0.69 & 2.23 \\
\cline { 2 - 5 } & GLMM-H & 0.98 & 0.66 & 1.45 \\
\hline \multirow{2}{*}{ Image 5 } & GMM & 0.96 & 0.77 & 2.12 \\
\cline { 2 - 5 } & GLMM-H & 0.97 & 0.73 & 2.02 \\
\hline
\end{tabular}

From Table6 it is observed that segmentation performance measures for all five images for segmentation method using generalized Laplace mixture model $\mathrm{s}$ much superior than those of segmentation methods based on GMM. Therefore the proposed segmentation method is superior than that based on GMM. This may be due to the platy kurtic nature of the image regions.Using the developed algorithm the image can also be reconstructed. There are several quality metrics for the performance measure of image quality. In this paper, the quality metrics considered are image fidelity, Mean Square 
Error, Signal to Noise Ratio and Image Quality Index. The computed values of image quality metrics are given in Table 7.

Table 7: Segmentation Quality Metrics

\begin{tabular}{|c|c|c|c|c|c|}
\hline \multirow[b]{2}{*}{ Image } & \multirow[t]{2}{*}{ Method } & \multicolumn{4}{|c|}{ Image Quality Metrics } \\
\hline & & $\begin{array}{l}\text { Image } \\
\text { Fidelity }\end{array}$ & $\begin{array}{c}\text { Mean } \\
\text { Square } \\
\text { Error }\end{array}$ & $\begin{array}{c}\text { Signal } \\
\text { to } \\
\text { Noise } \\
\text { Ratio }\end{array}$ & $\begin{array}{l}\text { Image } \\
\text { Quality } \\
\text { Index }\end{array}$ \\
\hline \multirow{2}{*}{ Image 1} & GMM & 0.99 & 0.88 & 0.24 & 1.0 \\
\hline & $\begin{array}{c}\text { GLMM- } \\
\text { H }\end{array}$ & 0.99 & 0.72 & 0.24 & 0.988 \\
\hline \multirow{2}{*}{ Image2 } & GMM & 0.99 & 0.82 & 2.01 & 0.99 \\
\hline & $\begin{array}{c}\text { GLMM- } \\
\mathrm{H}\end{array}$ & 0.99 & 0.65 & 2.03 & 0.99 \\
\hline \multirow{2}{*}{ Image3 } & GMM & 0.98 & 0.99 & 1.45 & 0.98 \\
\hline & $\begin{array}{c}\text { GLMM- } \\
\mathrm{H}\end{array}$ & 0.99 & 0.89 & 1.45 & 0.99 \\
\hline \multirow{2}{*}{ Image 4} & GMM & 0.99 & 0.99 & 2.23 & 1.0 \\
\hline & $\begin{array}{c}\text { GLMM- } \\
\mathrm{H}\end{array}$ & 0.99 & 0.92 & 2.69 & 1.0 \\
\hline \multirow{2}{*}{ Image 5} & GMM & 0.99 & 0.92 & 1.09 & 0.99 \\
\hline & $\begin{array}{c}\text { GLMM- } \\
\mathrm{H}\end{array}$ & 0.99 & 0.76 & 1.13 & 0.997 \\
\hline
\end{tabular}

The original images and reconstructed images using developed segmentation algorithm are presented in figure 4 .

\begin{tabular}{|c|c|c|}
\hline Image & Original Image & Reconstructed Image \\
\hline $\begin{array}{c}\text { Image } \\
1\end{array}$ & & \\
\hline $\begin{array}{c}\text { Image } \\
2\end{array}$ & & \\
\hline 3 \\
Image
\end{tabular}

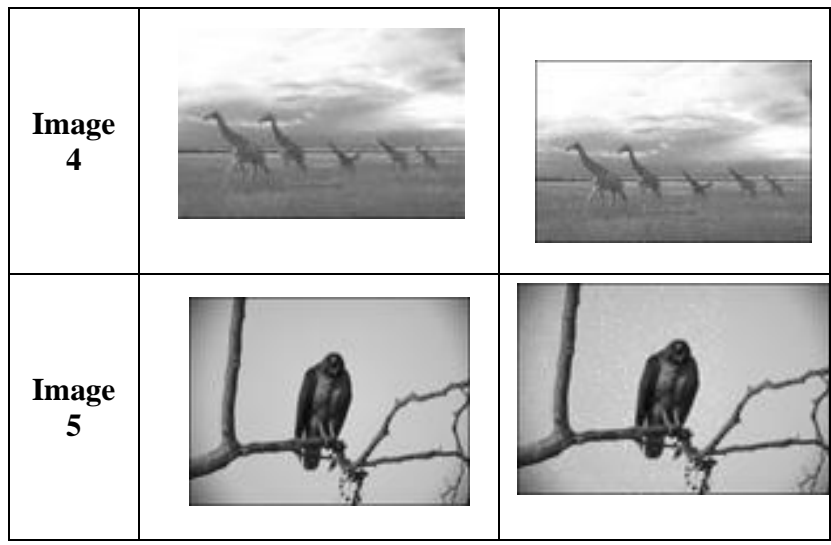

Fig 4: Original Image and Reconstructed Image

From table7 it is observed that the five images reconstructed using proposed method is more close to reality. The image fidelity value is almost same for both methods. However the mean square error is less for proposed method than that of method based on GMM. Similarly the Signal noise ratio for images 2,4 and 5 are bigger for proposed model than method based on GMM. For images 1 and 3 the signal noise ratio for both methods are same. Similar phenomenon is observed for image quality index. The proposed method incidentally provides an approach for dimension reduction in transmitting images i.e the estimated probability model of whole image with estimated parameters is sufficient for reconstruction of image.

\section{CONCLUSION}

In this paper an image segmentation technique using mixture of generalized Laplace distribution integrated with hierarchical clustering was designed and developed. Since in some images the pixel intensities of image regions are platy kurtic, the whole image is characterized by a k-component mixture of generalized Laplace probability model. The generalized Laplace probability model includes wide spectra of platy kurtic probability models for different values of shape parameter. The shape parameter of each image region is estimated using sample kurtosis of pixel intensities in image region. The other parameters of the model are estimated by deriving updated equations for the EM algorithm associated with generalized Laplace distribution. The initialization of parameters is carried using hierarchical clustering and moment method of estimation. Performance of proposed model is validated through conducting experimentation with five images randomly selected from Berkeley image database. The image segmentation performance measures such as GCE, PRI, and VOI are computed. From this experimentation it is observed that the deviation from GMM without affecting symmetry is highly effective in segmenting natural images such as bridge, bird, horse, giraffe and flying bird which are having platy kurtic image regions. It is also observed from the image quality metrics for the image retrievals computed with this five images in image reconstruction outperforms existing image segmentation algorithms when pixel intensities in each image region are having platy kurtic distribution. It is possible to extend this segmentation and image retrieval for color images by considering multivariate generalization of Laplace distribution which will be taken up elsewhere.

\section{REFERENCES}

[1] Srinivas Yerramalle And K.Srinivasa Rao (2007), "Unsupervised image classification using finite truncated 
Gaussian mixture model", Journal of Ultra Science for Physical Sciences, Vol.19, No.1, pp 107-114.

[2] Hua Yang; Jie Tian; Jia Yang (2000), "New medical image segmentation algorithm based on Gaussianmixture model", Proc. SPIE 4224, Biomedical Photonics and Optoelectronic Imaging.

[3] Yunjie Chen , Bo Zhao, Jianwei Zhang, Jin Wang and Yuhui Zheng, (2014), "An Improved Gaussian Mixture Model based on NonLocal Information for Brain MR Images Segmentation" International Journal of Signal Processing, Image Processing and Pattern Recognition Vol.7, No.4 pp.187-194

[4] Celia A. Z. Barcelos, Yunmei Chen, Fuhua Chen, (2014), "Soft Image Segmentation Based on the Mixture of Gaussians and the Phase-Transition Theory", Scientific Research Applied Mathematics,5, 2888-2898.

[5] ShanazAman, Farhat Afreen , Harapriya Sahoo , Chandana Patel , Rajan Jha, Ashim Saurav Implementing GMM-Based Hidden Markov (2015), "Random Field For Colour Image Segmentation", International Journal Of Engineering Sciences \& Research Technology ISSN: 2277-9655.

[6] M.Vamsi Krishna, Dr. P. V. G. D. Prasad Reddy, Dr.Y. Srinivas, Dr.S.S.Nayak(2015) "Bivariate Gaussian Mixture Model Based Segmentation for Effective Identification of Sclerosis in Brain MRI Images", International Journal of Engineering and Technical Research (IJETR) ISSN: 2321-0869, Volume - 3, Issue 1.

[7] K. Srinivasa Rao, M.Seshashayee, Ch.Satyanarayana, P.Srinivasa Rao, (2012), "Performance of Hybrid Imag e Segmentation Based On New Symmetric Mixture Model and Hierarchical Clustering", International Journal of Graphics and Image Processing, vol 2, issue 3.

[8] G.V.S. Rajkumar, K.Srinivasa Rao And P.Srinivasa Rao,(2011), "Image Segmentation Method Based On
Finite Doubly Truncated Bivariate Gaussian Mixture Model with Hierarchical Clustering”, IJCSI International Journal of Computer Science Issues, Vol. 8, Issue 4, No 2.

[9] Martin D. , Fowlkes C., D. Tal, and J.Malik (2001) “ A database of human segmented natural images and its application to evaluating segmentation algorithms and measuring ecological statistics," in proc. 8th Int. Conf. Computer vision, vol.2, pp.416-423.

[10] Johnson S.C(1967) "A Tutorial on Clustering Algorithms", http://home.dei.polimi.it/matteucc/Clustering/tutorial_ht $\mathrm{ml} /$ hierarchical.html

[11] Lei T. and Udupa J. (2003) "Performance evaluation of fini e normal mixture model-based image segmentation techniques," IEEE Transactions on Image Processing, vol. 12, no.10, pp. 1153-1169.

[12] Norman L.Johnson, Kortz and Balakrishnan(1994), "Continuous Univariate distributions"Volume-I, John Wiley and Sons Publications,Newyork.

[13] Srinivas Rao. K,C.V.S.R.Vijay Kumar, J.Lakshmi Narayana, (1997) " On a New Symmetrical Distribution ", Journal of Indian Society for Agricultural Statistics, Vol.50(1), pp 95-102.

[14] T. Yamazaki (1998), “ Introduction of EM algorithm into color image segmentation," in Proceedings of ICIRS'98, pp. $368-371$.

[15] Mclanchlan G. And Krishnan T(1997)., “ The EM Algorithm and Extensions", John Wiley and Sons, New York -1997.

[16] Xu L. and Jordan I., "On Convergence Properties of the EM Algorithme for Gaussian Mixture," Neural Computation, vol. 8, no. 1, pp.129-151, 1996. 